\title{
Xuebijing injection alleviates cytokine-induced inflammatory liver injury in CLP-induced septic rats through induction of suppressor of cytokine signaling 1
}

\author{
AILIN LI ${ }^{1 *}$, JING LI $^{2 *}$, YUHUA BAO ${ }^{1}$, DINGSHAN YUAN ${ }^{1}$ and ZHONGWEI HUANG ${ }^{1}$ \\ ${ }^{1}$ Department of Emergency Medicine, The Affiliated Hospital of Nantong University, Nantong, Jiangsu 226001, P.R. China; \\ ${ }^{2}$ Department of Genetics and Biochemistry, Clemson University, Clemson, SC 29634, USA
}

Received November 3, 2015; Accepted June 8, 2016

DOI: $10.3892 /$ etm.2016.3476

\begin{abstract}
Dysregulation of inflammatory cytokines and liver injury are associated with the pathogenesis of sepsis. Xuebijing injection, a Chinese herbal medicine, has been used in the treatment of sepsis and can contribute to the improvement of patients' health. However, the underlying molecular mechanisms are not yet clearly illuminated. In the present study, a septic rat model with liver injury was established by the cecal ligation and puncture (CLP) method. Histological alterations to the liver, activities of alanine aminotransferase (ALT) and aspartate aminotransferase (AST), levels of inflammatory cytokine secretion and the expression of suppressors of cytokine signaling 1 (SOCS-1) in the CLP model rats with and without Xuebijing treatment were determined. The results showed that Xuebijing injection ameliorated the pathological changes in liver tissues caused by sepsis, and reduced the sepsis-induced elevation in serum ALT and AST levels. Furthermore, Xuebijing injection markedly downregulated the expression of tumor necrosis factor $\alpha$ and interleukin (IL)-6,
\end{abstract}

Correspondence to: Dr Dingshan Yuan or Professor Zhongwei Huang, Department of Emergency Medicine, The Affiliated Hospital of Nantong University, 20 Xisi Road, Nantong, Jiangsu 226001, P.R. China

E-mail: dingshanyuan@sina.com

E-mail: tdfyhuangzw@163.com

${ }^{*}$ Contributed equally

Abbreviations: SOCS, suppressors of cytokine signaling; TNF- $\alpha$, tumor necrosis factor- $\alpha$; NF- $\kappa B$, nuclear factor $\kappa B$; IL-6, interleukin-6; IL-10, interleukin-10; SIRS, systemic inflammatory response syndrome; CLP, cecal ligation and puncture; ALT, alanine aminotransferase; AST, aspartate aminotransferase; RT-qPCR, reverse transcription-quantitative polymerase chain reaction; ELISA, enzyme-linked immunosorbent assay; JAK-STAT, janus kinase-signal transducer and activator of transcription; LPS-NF- $\kappa \mathrm{B}$, lipopolysaccharide-nuclear factor- $\mathrm{\kappa B}$

Key words: Xuebijing injection, sepsis, SOCS-1 and upregulated the expression of IL-10. More importantly, SOCS1 expression levels at the protein and mRNA levels were further increased by Xuebijing. These findings demonstrate that Xuebijing injection can significantly alleviate liver injury in CLP-induced septic rats via the regulation of inflammatory cytokine secretion and the promotion of SOCS1 expression. The protective effects of Xuebijing injection suggest its therapeutic potential in the treatment of CLP-induced liver injury.

\section{Introduction}

Sepsis is a serious systemic inflammatory response syndrome (SIRS) caused by infection with bacteria, fungi, viruses or parasites (1). The pathogenesis of sepsis is associated with disorder of whole-body inflammatory reaction networks (2). When the immune system becomes overactivated in response to an existing infection, the inflammatory response may lead to dysfunction of vital organs, such as the lung, heart, kidney and liver (3). The liver plays a critical role in the host defense mechanism (4), and is one of the organs most frequently affected by organ dysfunction in sepsis (5). The liver modulates inflammatory processes, and produces and releases high amounts of various cytokines (6), which play an important role in the regulation and transduction of inflammatory signals (7). During sepsis, pro-inflammatory cytokines, including tumor necrosis factor (TNF)- $\alpha$ and interleukin (IL)-6, have a crucial effect on the initiation of the inflammatory process (8). Anti-inflammatory cytokines, such as IL-10, are also found to be extensively released in septic patients (9). Moreover, cytokines mediate and amplify inflammatory signals through the Janus kinase-signal transducer and activator of transcription JAK-STAT pathway in a number of diseases (10). A recent study has revealed that suppressors of cytokine signaling (SOCS) are able to suppress cytokine-mediated inflammatory signaling as feedback regulators in the JAK-STAT pathway (11). SOCS-1, a member of the SOCS family, has been experimentally demonstrated to be effective in reducing the inflammatory response in inflammatory diseases $(12,13)$. Mice deficient in SOCS-1 have exhibited liver degeneration and lymphoid deficiencies (14). Furthermore, a previous study has shown that sepsis induces SOCS-1 expression in rat liver and 
muscle (15), and it has been recognized that SOCS1 plays an important role in LPS-induced liver injury. Therefore, SOCS-1 may be involved in the pathogenesis of liver injury in cecal ligation and puncture (CLP)-induced septic rats.

Xuebijing injection is a newly developed Chinese herbal injection consisting of Chuangxiong Rhizoma (the dried rhizome of Ligusticum chuanxiong Hort.), Paeoniae Radix Rubra (the dried roots of Paeonia lactiflora Pall.), Salviae Miltiorrhizae Radix et Rhizoma (the dried roots and rhizome of Salvia miltiorrhiza Bge.) and Carthami Flos (the dried flower of Carthamus tinctorius L.). It has anti-inflammatory and anti-endotoxic effects in the treatment of sepsis (16). Experimental and clinical studies have revealed that Xuebijing injection is effective for the treatment of SIRS, pyemia and multiple organ dysfunction syndrome (17-19). In addition, Xuebijing injection has been formally approved by the State Food and Drug Administration of China for use in clinical practice and is widely used to treat sepsis in China. However, the underlying molecular mechanisms of Xuebijing injection remain unclear. Thus, the aim of the present study was to investigate the effect of Xuebijing injection on liver injury in CLP-induced septic rats and to explore the underlying mechanism. In particular, the study aimed to focus on the effect of Xuebijing injection on hepatic inflammatory cytokine secretion and SOCS1 expression in rats with sepsis-induced liver injury.

\section{Materials and methods}

Chemicals and antibodies. Xuebijing injection was supplied by Tianjin Chase Sun Pharmaceutical Co., Ltd. (Tianjin, China; lot no. Z20070410). Antibodies targeting SOCS-1 (sc-9021) and $\beta$-actin (sc-130656) were purchased from Santa Cruz Biotechnology, Inc. (Dallas, TX, USA). Horseradish peroxidase (HRP)-conjugated anti-rabbit IgG antibody (RPN4301) for the western blotting assay was purchased from Amersham (GE Healthcare Life Sciences, Piscataway, NJ, USA). Enzyme-linked immunosorbent assay (ELISA) kits for TNF- $\alpha$, IL-6 and IL-10 were purchased from R\&D Systems, Inc. (Minneapolis, MN, USA). The ABI StepOnePlus Real-Time PCR System was purchased from Applied Biosystems (Thermo Fisher Scientific, Inc., Waltham, MA, USA). All other chemicals used were of analytical grade.

Animals. Male Sprague-Dawley (SD) rats, weighing 240-280 g, were provided by the Experimental Animal Center of Nantong University (Nantong, China) and housed in a temperatureand humidity-controlled environment with a 12-h light-dark cycle. The animals had access to food and water ad libitum. The study protocol was approved by the ethics committee (Institutional Review Board) of Nantong University.

Animal model and treatment. A total of 72 rats were divided into three experimental groups: Control group, CLP group and Xuebijing injection-treated (XT) group. The rats in the latter group were treated with $10 \mathrm{mg} / \mathrm{kg}$ Xuebijing injection, intraperitoneally, i.p. Each group contained 24 rats. The septic rat model was induced by CLP as previously described (20). Briefly, all rats underwent fasting for $12 \mathrm{~h}$ and water deprivation for $6 \mathrm{~h}$ prior to undergoing CLP surgery. Following pentobarbital sodium anesthesia ( $45 \mathrm{mg} / \mathrm{kg}$, i.p.), the abdominal region was disinfected with iodophor and a $2-\mathrm{cm}$ incision was made, the cecum was then gently isolated with tight ligation and punctured twice with an 18-gauge needle. Thereafter, the cecum was repositioned, and the abdomen was subsequently closed. For the control group alone, rats underwent the same surgical procedures, but the cecum was neither ligated nor punctured. Saline $(50 \mathrm{ml} / \mathrm{kg}$ body weight) was administered subcutaneously to the rats for fluid resuscitation. For the TX group, rats received an intraperitoneal administration of Xuebijing injection (10 $\mathrm{ml} / \mathrm{kg}$ body weight) twice per day after CLP surgery for 3 consecutive days. Rats in the control and CLP groups were administered equal volumes of saline instead.

Alanine aminotransferase (ALT) and aspartate aminotransferase (AST) activity measurements. To measure ALT and AST activities, 5-ml blood samples were obtained from the aorta abdominalis of rats in different groups at 6 , 12, 24 and $24 \mathrm{~h}$ after CLP surgery. The ALT and AST activities were then measured using a Hitachi 7600-020 Automatic Analyzer (Hitachi High-Technologies Corporation, Tokyo, Japan).

ELISA analysis of TNF- $\alpha, I L-6$, and IL-10. Homogenized liver samples $(0.5-1 \mathrm{~g} ; 10 \%)$ from the different groups, sampled at 6, 12,24 and $24 \mathrm{~h}$ after CLP surgery, were centrifuged at $1,500 \mathrm{x} \mathrm{g}$ for $15 \mathrm{~min}$ at $4^{\circ} \mathrm{C}$ ) to obtain a supernatant. The amounts of TNF- $\alpha$, IL- 6 and IL-10 in the liver tissue homogenates were analyzed using commercial ELISA kits specific for rats according to the manufacturer's protocol. Cytokine levels are expressed in units of $\mathrm{pg} / \mathrm{ml}$.

Histological examination. The liver tissues from rats in each group were collected at 6 and $48 \mathrm{~h}$ after CLP surgery. The tissue samples were fixed in $10 \%$ formalin solution, embedded in paraffin, and sectioned. The tissue sections were then stained with hematoxylin and eosin reagent according to standard protocols and observed using light microscopy.

Reverse transcription-quantitative polymerase chain reaction $(R T-q P C R)$. To determine the mRNA level of SOCS-1, RT-qPCR analysis was performed. Briefly, liver tissues samples (50-100 $\mathrm{mg}$ ) were collected from rats in the various groups at $6,12,24$ and $48 \mathrm{~h}$ after surgery. Total RNA was then extracted from homogenized liver samples using TRIzol reagent (Gibco Life Technologies; Thermo Fisher Scientific, Inc.) according to the manufacturer's protocol. Total RNAs were treated with DNase I (Fermentas; Thermo Fisher Scientific, Inc.) to remove any contaminating DNA at $37^{\circ} \mathrm{C}$ for $30 \mathrm{~min}$, followed by deactivation at $65^{\circ} \mathrm{C}$ for $10 \mathrm{~min}$. The RNA was then reverse transcribed into cDNA using an M-MLV reverse transcription kit (Takara Biotechnology Co., Ltd., Dalian, China) according to the manufacturer's protocol with the ABI StepOnePlus Real-Time PCR system. Following the reverse transcription, fluorescence qPCR was performed using SYBR Green I dye (Bio-Rad Laboratories, Inc., Hercules, CA, USA) according to the manufacturer's protocol. The following primers were used: SOCS-1 forward, 5'-CCGCTCCCACTCTGATTA CC-3' and reverse, 5'-CCCGAAGCCATCTTCACG-3'; $\beta$-actin forward, 5'-CGGTTCCGATGCCCTGAGGCACTT-3' and 
reverse, 5'-CGTCACACTTCATGATGGAATTGA-3'. The samples for RT-qPCR analysis were evaluated using a single predominant peak as a quality control. The comparative $\mathrm{Ct}$ $\left(2^{-\Delta \Delta C}\right)$ method (21) was used to analyze the relative expression levels of SOCS1 mRNA, which were normalized to $\beta$-actin.

Western blot analysis. Western blot analysis was performed using a similar method to that previously described (22). Briefly, homogenized liver tissues from different groups, sampled at 12 and $48 \mathrm{~h}$, were centrifuged at $13,000 \mathrm{x} \mathrm{g}$ for $30 \mathrm{~min}$ at $4^{\circ} \mathrm{C}$ to generate a supernatant containing the extracted protein. The protein concentration was measured using a bicinchoninic acid (BCA) protein assay kit as previously described $(23,24)$. A $50 \mu \mathrm{g}$ portion of each sample was electrophoresed on polyacrylamide gel (10\%) and transferred onto a polyvinylidene difluoride membrane (EMD Millipore, Bedford, MA, USA). After blocking with blocking buffer, the blots were incubated $2 \mathrm{~h}$ at $4^{\circ} \mathrm{C}$ with diluted primary antibodies against SOCS-1 (1:200). The membrane was then washed three times in Tris-buffered saline with Tween-20 $(0.1 \%)$ and incubated with HRP-conjugated anti-rabbit IgG antibody (dilution, 1:2,500) for $1.5 \mathrm{~h}$ at room temperature. The membrane was again washed three times for 10 min each time, and finally the immunoreactive proteins were detected using an enhanced chemiluminescence Western blotting detection kit. The $\beta$-actin protein served as an internal control.

Statistical analysis. The data were analyzed using STATA version 10.0 (StataCorp LP, College Station, TX, USA). One-way analysis of variance (ANOVA) was used to analyze the significant differences among different groups. $\mathrm{P}<0.05$ was considered to indicate a statistically significant difference.

\section{Results}

Xuebijing injection ameliorates sepsis-induced histological liver injury in rats. Hematoxylin and eosin staining clearly revealed liver injury characterized by edema, ballooning degeneration, fatty degeneration, thrombosis and infiltration of inflammatory cells in the liver tissues at $6 \mathrm{~h}$ after CLP surgery (Fig. 1). When examined at $48 \mathrm{~h}$ after CLP surgery, hepatic cords were largely broken and all liver injury symptoms become much more severe. However, these histological alterations induced by CLP surgery were ameliorated by Xuebijing injection treatment (Fig. 1).

Xuebijing injection decreases ALT and AST levels in rats with septic liver injury. ALT and AST levels of rats from different groups were measured at 6,12,24 and $24 \mathrm{~h}$ after CLP surgery. Sepsis induced an increase of serum ALT and AST levels in rats in comparison with those in the control group $(\mathrm{P}<0.05)$, which was indicative of liver injury. However, as shown in Fig. 2, treatment with Xuebijing injection significantly decreased ALT and AST levels in this model $(\mathrm{P}<0.05)$.

Xuebijing injection regulates the secretion of inflammatory cytokines in CLP-induced septic rats. Sepsis is characterized by the uncontrollable release of pro-inflammatory and anti-inflammatory cytokines (25). In the present study, two pro-inflammatory cytokines, TNF- $\alpha$ and IL-6, and one

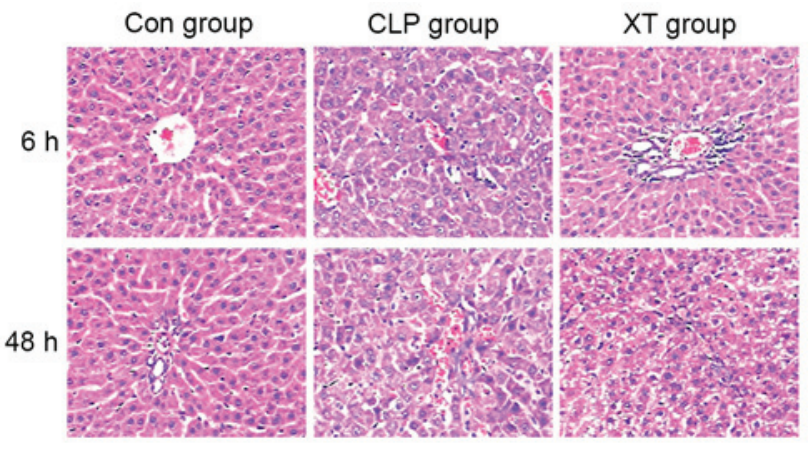

Figure 1. Effects of Xuebijing injection on CLP-induced histological liver injury in CLP-induced septic rats. Con group, control group; CLP group, cecal ligation and puncture group; XT group, CLP surgery plus Xuebijing injection treatment. Liver sections with hematoxylin and eosin staining (magnification, $\mathrm{x} 200$ ).
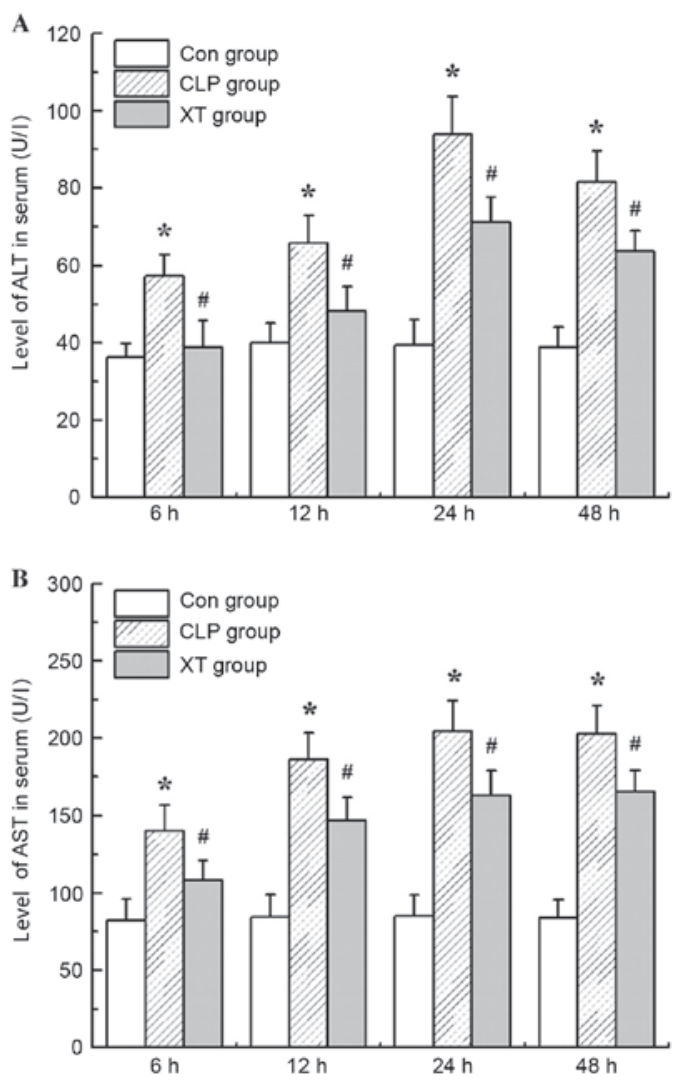

Figure 2. Effects of Xuebijing injection on the levels of (A) ALT and (B) AST in CLP-induced septic rats. ALT, alanine aminotransferase; AST, aspartate aminotransferase; Con group, control group; CLP group, cecal ligation and puncture group; XT group, CLP surgery plus Xuebijing injection treatment. Data are expressed as the mean \pm standard deviation $(n=6)$. ${ }^{*} \mathrm{P}<0.05$ vs. the Con group; ${ }^{\#} \mathrm{P}<0.05$ vs. the CLP group.

anti-inflammatory cytokine, IL-10 were detected at 6, 12, 24 and $24 \mathrm{~h}$ after CLP surgery using ELISA kits. The results in Fig. 3 show that hepatic TNF- $\alpha$, IL-6 and IL-10 levels were significantly elevated in CLP-induced septic rats in comparison with those in the control group $(\mathrm{P}<0.05)$. However, Xuebijing injection treatment significantly downregulated hepatic TNF- $\alpha$ and IL-6 levels, while upregulating hepatic IL-10 levels in CLP-induced septic rats $(\mathrm{P}<0.05$ for each). 

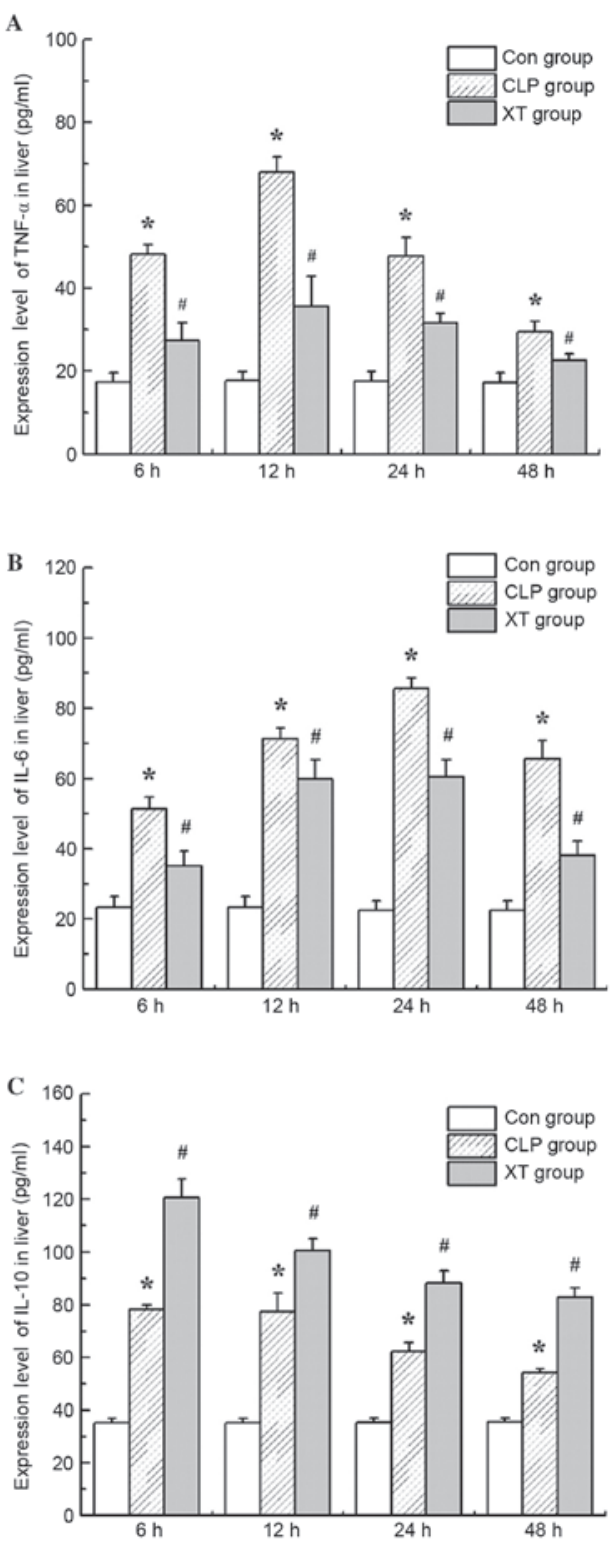

Figure 3. Effects of Xuebijing injection on the secretion of inflammatory cytokines in CLP-induced septic rats. Expression levels of (A) TNF- $\alpha$, (B) IL-6 and (C) IL-10 were determined. TNF, tumor necrosis factor; IL, interleukin; Con group, control group; CLP group, cecal ligation and puncture group; XT group, CLP surgery plus Xuebijing injection treatment. Values are expressed as the mean \pm standard deviation $(\mathrm{n}=6)$. ${ }^{*} \mathrm{P}<0.05$ vs. the Con group; ${ }^{\#} \mathrm{P}<0.05$ vs. the CLP group.

Xuebijing injection elevates the expression of SOCS-1 in $C L P$-induced septic rats. Numerous studies have found that SOCS proteins are negative-feedback regulators in the JAK-STAT pathway (11). In the present study, the expression of SOCS-1 was analyzed by RT-qPCR and western blotting. As shown in Fig. 4, elevated SOCS-1 mRNA and protein expression levels were observed in CLP-induced septic rats $(\mathrm{P}<0.05$ for mRNA), and the increases were further increased by the Xuebijing injection treatment (Fig. 4).

\section{Discussion}

In the present study, it was demonstrated that Xuebijing injection treatment attenuated liver injury induced by sepsis.
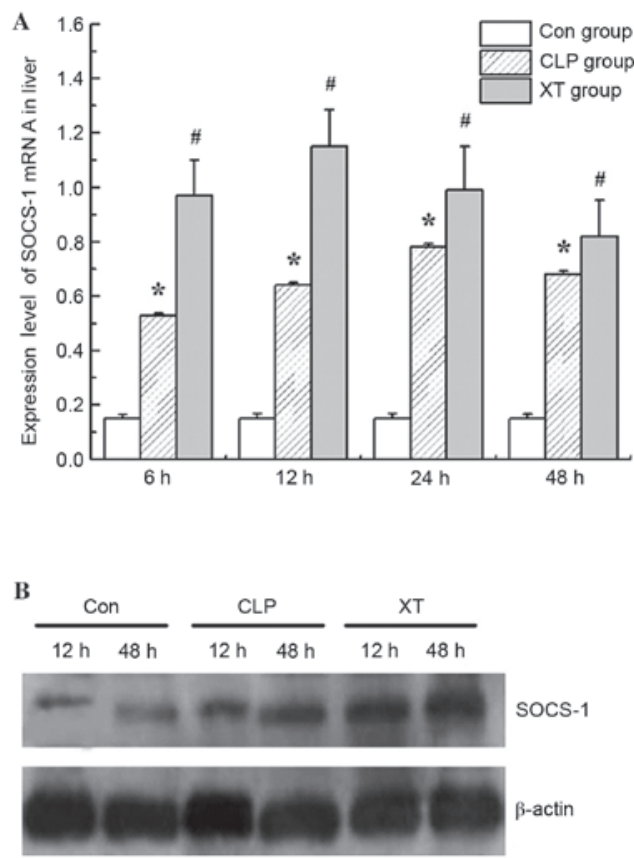

Figure 4. Effects of Xuebijing injection on the expression of SOCS-1 in CLP-induced septic rats. (A) SOCS-1 mRNA expression levels determined by reverse transcription-quantitative polymerase chain reaction. Values are expressed as the mean \pm standard deviation of 6 rats in each group. ${ }^{*} \mathrm{P}<0.05$ vs. the Con group; ${ }^{\#} \mathrm{P}<0.05$ vs. the CLP group. (B) A representative Western blot from three experiments is shown; $\beta$-actin was used as a loading control. SOCS, suppressors of cytokine signaling; Con group, control group; CLP group, cecal ligation and puncture surgery group; XT group, CLP surgery plus Xuebijing injection treatment.

Furthermore, the results showed that Xuebijing injection treatment downregulated TNF- $\alpha$ and IL-6 levels, and upregulated IL-10 levels in the livers of septic rats, which was associated with the expression of SOCS1. Therefore, the present study suggests that Xuebijing injection protects against sepsis-induced liver injury by regulating the secretion of inflammatory cytokines and increasing the expression of SOCS1 in the livers of septic rats.

A previous clinicopathological study reported that patients dying from sepsis typically had inflammation and necrosis in the liver; increased levels of markers of abnormal liver biochemistry, including ALT and AST, were also observed in septic patients prior to death (26). In the present study, CLP-induced sepsis caused an inflammatory response and damage in the liver, which was characterized both histologically and biochemically. The treatment with Xuebijing injection ameliorated the pathological changes in liver tissues caused by sepsis, and also decreased ALT and AST levels in the rats with septic liver injury.

Evidence indicates that inflammatory cytokines play an important role in sepsis; pro-inflammatory cytokines and anti-inflammatory cytokines counteract each other and reach an immunological equilibrium, which leads to various types of tissue damage and organ injury (8). In the present study, elevated TNF- $\alpha$, IL- 6 and IL-10 levels were observed in the livers of rats in the CLP group in comparison with those in the control group, which is consistent with previous studies $(27,28)$. Moreover, Xuebijing injection has been found to reduce the secretion of TNF- $\alpha$ and IL- 6 by Kupffer cells in livers of rats 
with heart stroke (29), and promote IL-10 expression in rabbits with oleic acid-induced acute lung injury (30). The present study found that Xuebijing injection significantly downregulated pro-inflammatory cytokine levels, specifically TNF- $\alpha$ and IL-6, while upregulating the levels of the anti-inflammatory cytokine IL-10 in rats with septic liver injury. These data suggest that the protective effects of Xuebijing injection on septic liver injury may occur via the regulation of the secretion of inflammatory cytokines.

Cytokine signaling induces SOCS1, a protein that can inhibit the signaling pathways that stimulated its production $(31,32)$. SOCS-1 is a negative-feedback regulator not only in the JAK-STAT pathway but also in the lipopolysaccharide-nuclear factor- $\kappa \mathrm{B}$ (LPS-NF- $\kappa$ B) signaling pathway (33). SOCS-1 has been found to inhibit cytokine signaling in all systems tested, including TNF- $\alpha$ and IL-6 (34). TNF- $\alpha$-mediated inflammatory signals can be transduced through the JAK-STAT pathway $(35,36)$. The results of the present study demonstrated that SOCS-1 expression was upregulated by Xuebijing injection, which corresponded with its relieving effects on inflammatory response and liver injury. Collectively, those results indicate that Xuebijing injection ameliorates liver injury in CLP-induced septic rats by promoting SOCS-1 expression at the protein and mRNA levels.

In summary, the present study demonstrated that Xuebijing injection can effectively alleviate liver injury in a rat model of CLP-induced sepsis. The protective effects of Xuebijing injection on septic liver injury are associated with the regulation of inflammatory cytokine secretions and the promotion of SOCS1 expression. This study provides valuable insights into the role of Xuebijing injection in septic liver injury, and reveals its therapeutic potential in CLP-induced liver injury treatment.

\section{Acknowledgements}

This study was supported by grants from the China Postdoctoral Science Foundation (2013M541707), Jiangsu Postdoctoral Science Foundation, Natural Science Fund Project in Zhejiang Province (Y2100917) and Nantong Science and Technology Project (HS149095, HS149137 and HS2014075).

\section{References}

1. Jones AE and Puskarich MA: The surviving sepsis campaign guidelines 2012: Update for emergency physicians. Ann Emerg Med 63: 35-47, 2014.

2. Hotchkiss RS and Karl IE: The pathophysiology and treatment of sepsis. N Engl J Med 348: 138-150, 2003.

3. Abraham E and Singer M: Mechanisms of sepsis-induced organ dysfunction. Crit Care Med 35: 2408-2416, 2007.

4. Spapen H: Liver perfusion in sepsis, septic shock, and multiorgan failure. Anat Rec (Hoboken) 291: 714-720, 2008.

5. Regel G, Grotz M, Weltner T, Sturm JA and Tscherne H: Pattern of organ failure following severe trauma. World J Surg 20: 422-429, 1996.

6. Dhainaut JF, Marin N, Mignon A and Vinsonneau C: Hepatic response to sepsis: Interaction between coagulation and inflammatory processes. Crit Care Med 29 (Suppl 7): S42-S47, 2001.

7. Zhang JM and An J: Cytokines, inflammation and pain. Int Anesthesiol Clin 45: 27-37, 2007.

8. Schulte W, Bernhagen J and Bucala R: Cytokines in sepsis: Potent immunoregulators and potential therapeutic targets-an updated view. Mediators Inflamm 2013: 165974, 2013.

9. Friedman G, Jankowski S, Marchant A, Goldman M, Kahn RJ and Vincent JL: Blood interleukin 10 levels parallel the severity of septic shock. J Crit Care 12: 183-187, 1997.
10. Villarino AV,Kanno Y,Ferdinand JR and O'Shea JJ: Mechanisms of Jak/STAT signaling in immunity and disease. J Immunol 194: 21-27, 2015

11. Alexander WS: Suppressors of cytokine signalling (SOCS) in the immune system. Nat Rev Immunol 2: 410-416, 2002.

12. Yamana J, Yamamura M, Okamoto A, Aita T, Iwahashi M, Sunahori K and Makino H: Resistance to IL-10 inhibition of interferon gamma production and expression of suppressor of cytokine signaling 1 in CD4+T cells from patients with rheumatoid arthritis. Arthritis Res Ther 6: R567-R577, 2004.

13. Egan PJ, Lawlor KE, Alexander WS and Wicks IP: Suppressor of cytokine signaling-1 regulates acute inflammatory arthritis and T cell activation. J Clin Invest 111: 915-924, 2003.

14. Starr R, Metcalf D, Elefanty AG, Brysha M, Willson TA, Nicola NA, Hilton DJ and Alexander WS: Liver degeneration and lymphoid deficiencies in mice lacking suppressor of cytokine signaling-1. Proc Natl Acad Sci USA 95: 14395-14399, 1998.

15. Johnson TS, O'Leary M, Justice SK, Maamra M, Zarkesh-Esfahani SH, Furlanetto R, Preedy VR, Hinds CJ, El Nahas AM and Ross RJ: Differential expression of suppressors of cytokine signalling genes in response to nutrition and growth hormone in the septic rat. J Endocrinol 169: 409-415, 2001.

16. Chen S, Dai G, Hu J, Rong A, Lv J, Su L and Wu X: Discovery of Xuebijing injection exhibiting protective efficacy on sepsis by inhibiting the expression of HMGB1 in septic rat model designed by cecal ligation and puncture. Am J Ther Aug 25, 2015 (Epub ahead of print).

17. Yin Q and Li C: Treatment effects of Xuebijing injection in severe septic patients with disseminated intravascular coagulation. Evid Based Complement Alternat Med 2014: 949254, 2014.

18. He XD, Wang Y, Wu Q, Wang HX, Chen ZD, Zheng RS, Wang ZS Wang JB and Yang Y: Xuebijing protects rats from sepsis challenged with Acinetobacter baumannii by promoting annexin A1 expression and inhibiting proinflammatory cytokines secretion. Evid Based Complement Alternat Med 2013: 804940, 2013.

19. Liu MW, Wang YH, Qian CY and Li H: Xuebijing exerts protective effects on lung permeability leakage and lung injury by upregulating Toll-interacting protein expression in rats with sepsis. Int J Mol Med 34: 1492-1504, 2014.

20. Rittirsch D, Huber-Lang MS, Flierl MA and Ward PA: Immunodesign of experimental sepsis by cecal ligation and puncture. Nat Protoc 4: 31-36, 2009.

21. Livak KJ and Schmittgen TD: Analysis of relative gene expression data using real-time quantitative PCR and the 2(-Delta Delta C(T)) method. Methods 25: 402-408, 2001.

22. Yu B, Xu P, Zhao Z, Cai J, Sternberg P and Chen Y: Subcellular distribution and activity of mechanistic target of rapamycin in aged retinal pigment epithelium. Invest Ophthalmol Vis Sci 55: 8638-8650, 2014.

23. Yang Y, Dou SX, Ren H, Wang PY, Zhang XD, Qian M, Pan BY and Xi XG: Evidence for a functional dimeric form of the PcrA helicase in DNA unwinding. Nucleic Acids Res 36: 1976-1989, 2008.

24. Yang Y, Dou SX, Xu YN, Bazeille N, Wang PY, Rigolet P, $\mathrm{Xu} \mathrm{HQ}$ and Xi XG: Kinetic mechanism of DNA unwinding by the BLM helicase core and molecular basis for its low processivity. Biochemistry 49: 656-668, 2010.

25. Adrie $\mathrm{C}$ and Pinsky MR: The inflammatory balance in human sepsis. Intensive Care Med 26: 364-375, 2000.

26. Koskinas J, Gomatos IP, Tiniakos DG, Memos N, Boutsikou M, Garatzioti A, Archimandritis A and Betrosian A: Liver histology in ICU patients dying from sepsis: A clinico-pathological study. World J Gastroenterol 14: 1389-1393, 2008.

27. Zhu W, Bao R, Fan X, Tao T, Zhu J, Wang J, Li J, Bo L and Deng X: PD-L1 blockade attenuated sepsis-induced liver injury in a mouse cecal ligation and puncture model. Mediat Inflamm 2013: 361501, 2013.

28. Zhang H, Wang W, Fang H, Yang Y, Li X, He J, Jiang X, Wang W, Liu S, Hu J, et al: GSK-3 $\beta$ inhibition attenuates CLP-induced liver injury by reducing inflammation and hepatic cell apoptosis. Mediators Inflamm 2014: 629507, 2014.

29. Chen Y, Tong H, Zhang X, Tang L, Pan Z, Liu Z, Duan P and Su L: Xuebijing injection alleviates liver injury by inhibiting secretory function of Kupffer cells in heat stroke rats. J Tradit Chin Med 33: 243-249, 2013.

30. Wang Y, Ji M, Wang L, Chen L and Li J: Xuebijing injection improves the respiratory function in rabbits with oleic acid-induced acute lung injury by inhibiting IL-6 expression and promoting IL-10 expression at the protein and mRNA levels. Exp Ther Med 8: 1593-1598, 2014. 
31. Chung CS, Chen Y, Grutkoski PS, Doughty L and Ayala A: SOCS-1 is a central mediator of steroid-increased thymocyte apoptosis and decreased survival following sepsis. Apoptosis 12: 1143-1153, 2007.

32. Davey G, Heath W and Starr R: SOCS1: A potent and multifaceted regulator of cytokines and cell-mediated inflammation. Tissue Antigens 67: 1-9, 2006.

33. Shuai K and Liu B: Regulation of JAK-STAT signalling in the immune system. Nat Rev Immunol 3: 900-911, 2003.

34. Fujimoto M and Naka T: Regulation of cytokine signaling by SOCS family molecules. Trends Immunol 24: 659-666, 2003.
35. Guo D, Dunbar JD, Yang CH, Pfeffer LM and Donner DB: Induction of Jak/STAT signaling by activation of the type 1 TNF receptor. J Immunol 160: 2742-2750, 1998.

36. Miscia S, Marchisio M, Grilli A, Di Valerio V, Centurione L, Sabatino G, Garaci F, Zauli G, Bonvini E and Di Baldassarre A: Tumor necrosis factor alpha (TNF-alpha) activates Jak1/Stat3-Stat5B signaling through TNFR-1 in human B cells. Cell Growth Differ 13: 13-18, 2002. 